\title{
Properties of Padovan Sequence
}

\author{
Dr. R. Sivaraman ${ }^{1}$ \\ ${ }^{1}$ Associate Professor, Department of Mathematics, D. G. Vaishnav College, Chennai, IndiaNational Awardee for \\ Popularizing Mathematics among masses \\ rsivaraman1729@yahoo.co.in
}

Article History: Received: 11 January 2021; Accepted: 27 February 2021; Published online: 5 April 2021

\begin{abstract}
Among several types of famous sequences that exist in all branches of mathematics, Padovan sequence is one of such sequences possessing amusing properties. In this paper, we shall discuss about Padovan Sequence and determine a special number called Plastic number. We also derive the ratio of its $(n+1)$ st term to the $n$th term as $n$ is very large, which is called "Limiting Ratio". Finally we discuss the geometrical interpretation of the terms of Padovan sequence.

Keywords: Padovan Sequence, Recurrence Relation, Characteristic Equation, Plastic Number, Limiting Ratio.

\section{Introduction}

Padovan Sequence is named after British Polymath Richard Padovan who became fascinated with the works of Dutch monk and architect Hans van der Laan to the extent that he wrote a book about him titled "Dom Hans van der Laan: modern primitive" in the year 1989. In this book Richard Padovan while mentioning works of Hans van der Laan attributed an interesting sequence of numbers which had striking resemblance with the most famous Fibonacci numbers. Later, British mathematician and science popularizer Ian Stewart while describing the same sequence in one of his books called the sequence as Padovan Sequence and the name remained thereafter. Though Padovan sequence resembles Fibonacci sequence, it differs quite significantly from Fibonacci numbers especially in the case of dealing with limiting ratio.
\end{abstract}

\section{Definition}

Let $P(1)=P(2)=P(3)=1$ and $P(n+3)=P(n+1)+P(n), \quad n \geq 1$ (2.1). The sequence of terms defined by $P(n)$ for $n=1,2,3,4,5, \ldots$ is called Padovan Sequence.

We notice that the first three terms of the sequence are 1 and from the fourth term, each term is the sum of one but previous two terms. That is, fourth term is sum of second and first terms, fifth term is sum of third and second terms and so on. With this convention, the first few terms of the Padovan sequence (with help of (2.1)) are given by $1,1,1,2,2,3,4,5,7,9,12,16,21,28,37,49,65,86,114,151,200, \ldots$. (2.2)

\section{Characteristic Equation}

From the recurrence relation used for generating Padovan sequence, we can obtain the Characteristic Equation for Padovan Sequence.

From definition (2.1), we get $P(n+3)=P(n+1)+P(n)$.

The auxiliary equation considering to this recurrence relation is $m^{3}-m-1=0$ (3.1) .

Equation (3.1) is called Characteristic Equation of Padovan sequence. We will try to determine the real roots of the Characteristic equation.

\subsection{Roots of the Characteristic Equation}

Being a cubic equation in $\mathrm{m}$, there is no straight forward method to determine the real roots of (3.1). First, we determine the number of real roots of the Characteristic equation.

If we consider $f(m)=m^{3}-m-1$ then there is only one sign change in $f(m)$. Hence, there must be exactly one positive real root for the characteristic equation.

\subsection{Determining the Root}

We try to determine that particular positive root. For this, we can consider Newton - Raphson Method.

If $x_{0}$ is the initial approximation lying in $[a, b]$ then the root of given equation can be determined from the successive iterates given by $x_{n+1}=x_{n}-\frac{f\left(x_{n}\right)}{f^{\prime}\left(x_{n}\right)}, n=0,1,2,3,4, \ldots$ 
We first notice that $f(1)=-1<0, f(2)=5>0$. Hence the root should lie between 1 and 2 . Since $|f(1)|<|f(2)|$, let us assume initial approximation to the root to be $x_{0}=1$.

Using (3.2), we have $x_{n+1}=x_{n}-\frac{x_{n}{ }^{3}-x_{n}-1}{3 x_{n}{ }^{2}-1}$

First Iteration: For $n=0$, we get $x_{1}=1-\frac{(-1)}{2}=1.5$

Second Iteration: For $n=1$, we get $x_{2}=1.5-\frac{0.875}{5.75}=1.34782$

Third Iteration: For $n=2$, we get $x_{3}=1.34782-\frac{0.100655}{4.44985}=1.3252$

Fourth Iteration: For $n=3$, we get $x_{4}=1.3252-\frac{0.0020566}{4.26846}=1.324718$

Fifth Iteration: For $n=4$, we get $x_{5}=1.324718-\frac{0.00000018233}{4.26463333}=1.3247179$

We can stop our iteration process here and conclude that the positive root of the characteristic equation (3.1) is 1.32471 approximately.

The number 1.32471 which happens to be root of the characteristic equation pertaining to the recurrence relation of Padovan sequence is called "Plastic Number".

\subsection{Graphical Verification}

In Figure 1, we have graph of the function $f(x)=x^{3}-x-1$.

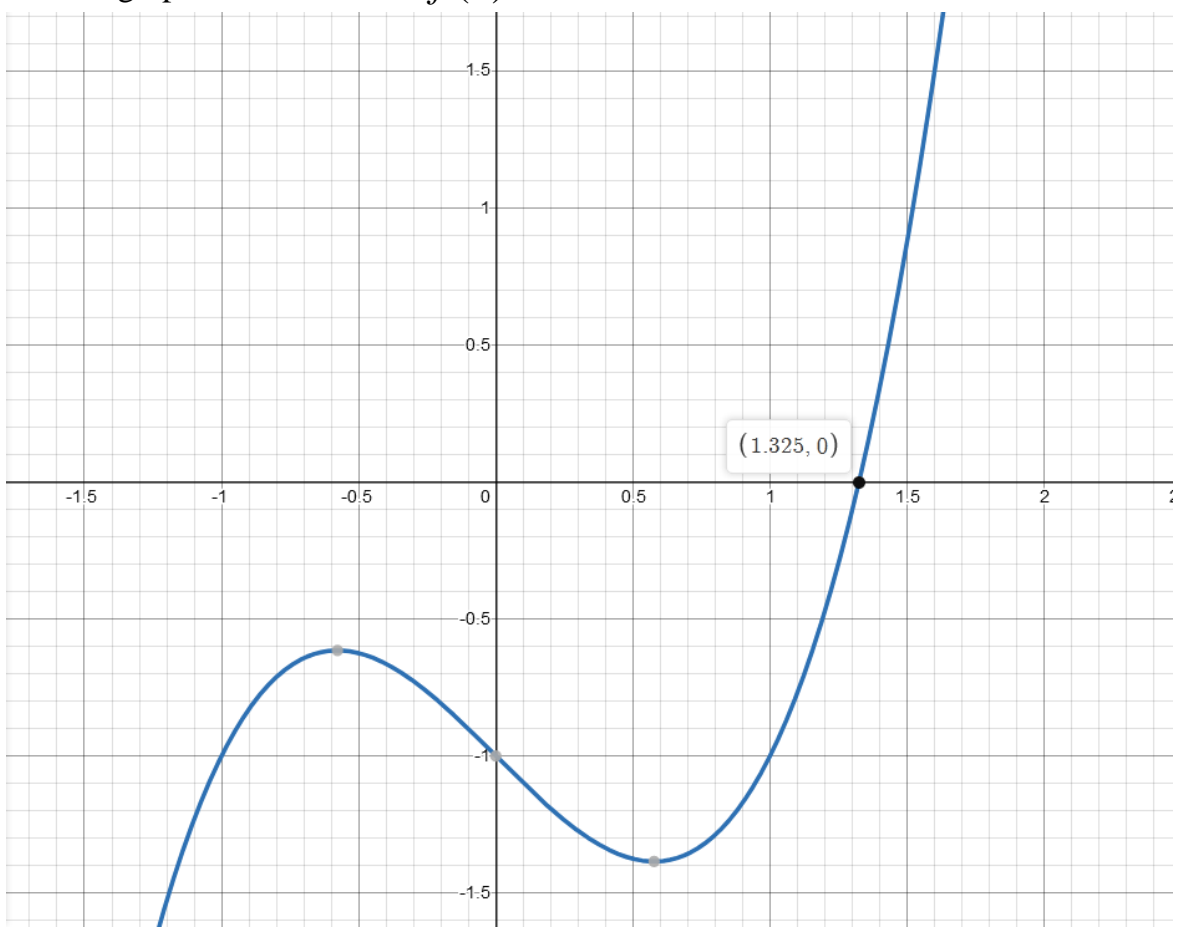

Figure 1: Graph of $f(x)=x^{3}-x-1$

We notice that the graph of the function crosses the $\mathrm{X}$ - axis at approximately 1.3247 which is the plastic number we obtained in section 3.2 through Newton - Raphson Method. This verifies the calculation of the plastic number, the root of the characteristic equation graphically.

\section{Limiting Ratio of Padovan Sequence}


In this section, we try to obtain the ratio of $(n+1)$ st term to the $n$th term of terms in the Padovan sequence as $n \rightarrow \infty$. We define such ratio as Limiting Ratio.

If we assume the limiting ratio as $\tau$, then by definition, as $n \rightarrow \infty, \frac{P(n+1)}{P(n)}=\tau$ (4.1)

Similarly, as $n \rightarrow \infty, \frac{P(n+3)}{P(n)}=\frac{P(n+3)}{P(n+2)} \times \frac{P(n+2)}{P(n+1)} \times \frac{P(n+1)}{P(n)}=\tau \times \tau \times \tau=\tau^{3}$

Thus, from (2.1), we have $P(n+3)=P(n+1)+P(n)$. Dividing by $P(n)$ we have $\frac{P(n+3)}{P(n)}=\frac{P(n+1)}{P(n)}+1$

Now considering the limit as $n \rightarrow \infty$, and using (4.1), (4.2), we get $\tau^{3}=\tau+1$ (4.4)

But we see that equation (4.4) is exactly same as the characteristic equation that we obtained in (3.1). Hence, the only positive root of (4.4) is given by the plastic number $\tau=1.32471$.

We thus have proved that the limiting ratio of terms of Padovan sequence is the plastic number which is approximately 1.32471

\section{Visualization of Terms of Padovan Sequence}

From (2.2), the terms of the Padovan sequence are given by $1,1,1,2,2,3,4,5,7,9,12,16,21,28,37$, . .

If we consider these numbers as sides of equilateral triangles and construct such triangles in spiral fashion oriented counter-clockwise, then we get Figure 2 shown below:

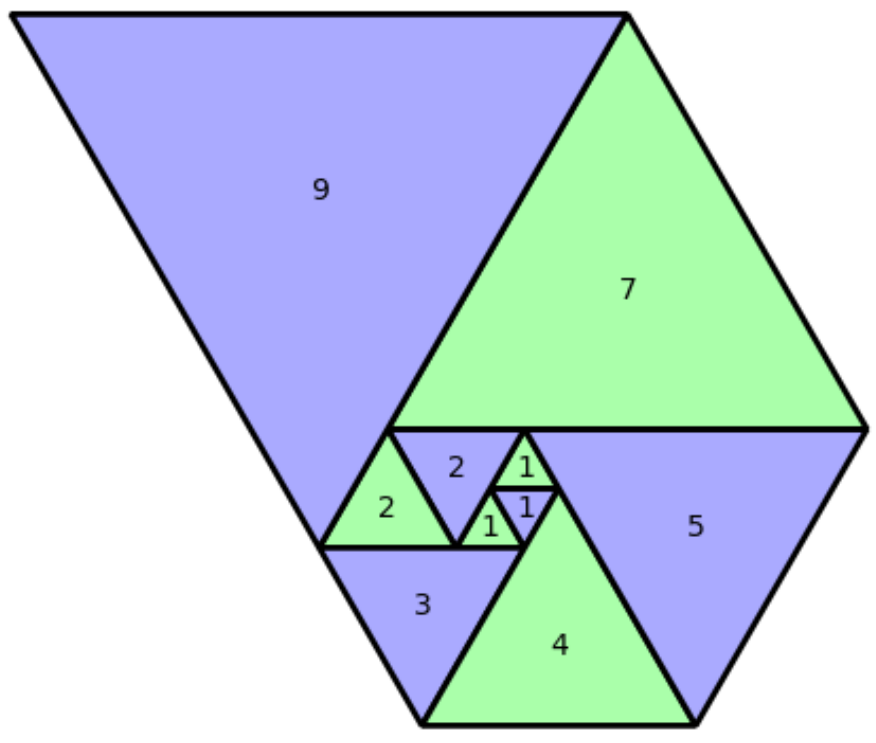

Figure 2: Spiral Triangles of Terms of Padovan Sequence

\section{Conclusion}

One of the fascinating sequences in mathematics called Padovan sequence is introduced in this paper. Using the recurrence relation obtained from the definition of the sequence, we determined the characteristic equation whose only positive real root is calculated using Newton - Raphson method and found to be a real number 1.32471 approximately. This number is called Plastic Number. We proved that the limiting ratio, that is ratio of $(n+1)$ st term to the $n$th term as $n$ is very large, of terms of the Padovan sequence is the plastic number, the root of the characteristic equation. Thus, we see that the plastic number for Padovan sequence plays the same role as that of Golden Ratio does for Fibonacci sequence. We verified the calculation of plastic number using Figure 1 obtained using Desmos Online Graphing Calculator. We know that the Fibonacci numbers produces rectangles stacked upon continuously. Similarly when we try to visualize the terms of the Padovan sequence, we got spiraling equilateral triangles as shown in Figure 2. Thus, the Padovan sequence exhibits almost the same characteristics as that of its cousin sequence Fibonacci sequence, leading to a new limiting ratio called plastic number. We can easily generalize this sequence and study the behaviour of such generalized Padovan sequences.

\section{REFERENCES}


1. Richard Padovan, Dom Hans van der Laan: modern primitive: Architectura \& Natura Press, ISBN 9789071570407.

2. Ian Stewart, Math hysteria: fun and games with mathematics, Oxford University Press, (2004), p. 87.

3. Gogin, N.D., Myllari, A.A. The Fibonacci-Padovan sequence and MacWilliams transform matrices. Program Comput Soft, 33, 74-79 (2007).

4. R. Sivaraman, Exploring Metallic Ratios, Mathematics and Statistics, Horizon Research Publications, Volume 8, Issue 4, (2020), pp. 388 - 391.

5. R. Sivaraman, Generalized Lucas, Fibonacci Sequences and Matrices, Purakala, Volume 31, Issue 18, April 2020, pp. $509-515$.

6. Graham, R.L., Knuth, D.E., and Patashnik, O., Concrete Mathematics, Addison-Wesley, 1994. 